\title{
USE OF WHITE WALLS IN A PHOTOMETRIC LABORATORY.
}

By Edward P. Hyde.

It is usually considered by photometricians that one of the essential requisites for accurate work in photometry is that the walls of the photometer room be made as nearly "dead black" as possible. It is surprising how long this tradition of photometry has persisted, particularly as it necessitates the exclusive use of a room for photometric measurements, since a room blackened in the customary manner is unfit for any other work. Perhaps it is due to this fact that one so often finds the photometric laboratory in a small room in the basement or garret, or some other place that is unsuited for any other purpose. In all open-flame work, however, much larger errors may result from poor ventilation in a small room than from light reflected from white walls of a larger room, if a proper set of screening diaphragms is used.

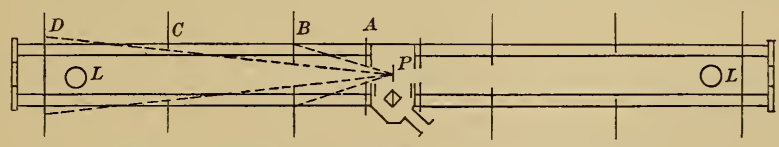

Fig. 1.-Horizontal section through center of photometer screen.

So far as I know nothing has been published as to the magnitude of the error due to white walls when the photometer is protected by black diaphragms, although this arrangement is at present in use to some extent in the photometric laboratory of the Physikalisch-Technische Reichsanstalt of Germany.

In the photometric laboratory of the Bureau of Standards the walls of one of the rooms, which is about 29 feet long, 19 feet wide, and 12 feet high, were left white for a year after the construction of the building. Recently they have been given a light terra-cotta finish for decorative purposes, but the following measurements were made while the walls were still white. The photometer is placed near the middle of the room and is supplied with a set of diaphragms covered with black velvet. These diaphragms may be so arranged for any position on the bar that no light, except that reflected from the lamps under test or from the diaphragms themselves can reach the photometer 2214-No. 3-05-9 
screen, as shown by the accompanying sketches. Fig. 1 is a horizontal section of the photometer through the center of the LummerBrodhun sight-box, showing the arrangement of diaphragms $A, B, C$, and $D$. Elevations of these diaphragms perpendicular to the axis of the photometer are given in fig. 2. By reference to fig. 1 it is seen that an eye placed at $P$ could see nothing but black velvet diaphragms and the lamps under test. Conversely, no light could reach the screen except from the lamps or from the diaphragms.

In case a Bunsen or Leeson disk is used, extra precautions would have to be taken to prevent light from penetrating to the screen from the front of the box. It is essential that this condition be fulfilled if the walls are to be left white, as any reflected light reaching the screen directly would materially affect the result. Furthermore, it is desirable that all outside light be excluded by the use of dark curtains, as under these circumstances the only light that can reach the photometer screen, apart from the direct light from the lamps, is that part of the light from the two lamps which is reflected by the walls back to the lamps and the diaphragms, and thence to the photometer.
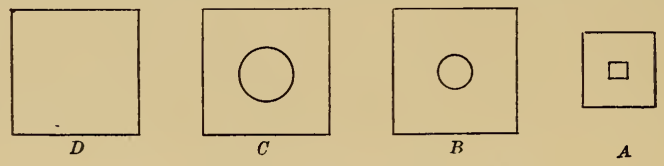

FIG. 2.-Front elevation of diaphragms.

A

In order to determine the magnitude of the errors resulting from this reflected light from the white walls of the photometer room of the Bureau of Standards, the measurements described below were made. The results of these measurements show conclusively that the error is entirely negligible.

Before giving the results of the measurements it may be well to say a word or two in regard to the method of measurement employed at the Bureau in the comparison of incandescent lamp standards. On the carriage on the right side of the photometer is placed a well seasoned incandescent lamp, though not ordinarily a standard. This lamp is raised to such a voltage that its color is approximately the same as the color of the lamps to be compared on the other side of the photometer, and it is kept at this voltage throughout the test. The carriage on which this comparison lamp is mounted is then rigidly connected by means of adjustable links to the carriage on which the photometer screen is mounted, and at a suitable distance to produce the desired illumination of the screen. The standard is then mounted on the carriage on the left side of the photometer, and is placed at such a position as to give a balance with the photometer at approximately the middle of the bar. The standard is brought to the proper 
voltage and several readings are taken. The lamps to be standardized are then mounted successively on the same carriage and readings are made. At the end the standard is again measured. If the lamps that are compared with the standard are of the same nominal candle-power as the standard, the left-hand carriage is kept at the same position on the bar. If the candle-power is different, the left-hand carriage is placed so that the balance comes at about the same place on the bar.

This method of measurement, which is a substitution method, has many advantages in accurate work. It eliminates all dissymmetry on the two sides of the photometer, and thus avoids the necessity of reversing the photometer screen.

In making the measurements of the effect of reflection from the walls the right side of the photometer was entirely protected from reflected light from the walls by large screens, which nearly surrounded that half of the photometer bar, so that when the left side of the Lummer-Brodhun sight-box was closed by a shutter no illumination was visible in the eyepiece. The velvet-covered diaphragms and the lamp on the left side of the photometer, on the contrary, were left exposed to the reflected light from the walls, as in the ordinary method of measurement, except that the lamp on that side was not burning. Therefore the only light that penetrated to the screen was that which, reflected from the walls, fell upon the lamp and black velvet diaphragms and was reflected by them to the screen.

In order that the walls should be illuminated to the same extent as in the ordinary measurements, a $16 \mathrm{c}$. p. lamp was burned near the position of the comparison lamp, and another lamp was burned near the position of the standard lamp, but so shielded in each case that no direct light could reach the photometer. The lamp on the left near the unburned standard was changed from time to time from a $32 \mathrm{c} . \mathrm{p}$. lamp to a $16 \mathrm{c}$. p. lamp, depending upon whether a 32 c. p. unburned lamp was in the left socket at the distance from the screen at which a 32 c. p. lamp is usually placed or a 16 c. p. lamp was in the socket at the position used with a $16 \mathrm{c}$. p. lamp. These precautions were taken in order to secure as nearly as possible working conditions.

Under these circumstances it was perfectly plain, on looking into the eyepiece of the photometer, that the left side of the sight-field was slightly illuminated, and it only remained to measure this illumination under the different conditions likely to occur. To this end a 1 c. p. lamp, which had been placed on the carriage on the right in the position of the comparison lamp, was brought to incandescence, and the light from this lamp was cut down by absorption strips of smoked glass having known coefficients of transmission. Thus successive absorption strips were added until a balance was obtained, and the 
number of strips and the positions of the three carriages were recorded. This method of procedure was followed through a great number of measurements, the conditions being changed from time to time until all the conditions that are actually encountered were included in order to determine whether any error due to the walls could possibly be introduced in any comparisons that are ever made. What was actually measured each time of course was the illumination of the screen due to the reflected light, and it was merely a matter of computation to determine what the candle-power at any distance would be in order to produce the illumination found.

I shall not give the results of all the measurements that were made, but merely indicate the results for two typical cases which show clearly the order of magnitude of the effect. First, from the direct measurement of the illumination that was produced when a 32 c. p. standard (not burning) was placed at the position at which a $32 \mathrm{c}$. p. lamp is generally placed in standard measurements, and another 32 c. p. lamp was illuminating the walls, calculation showed that the illumination on the screen, due to the reflection from the walls, was that which would be produced by a source of 0.003 candle at the position of the $32 \mathrm{c}$. p. standard. In a similar way the correction to a $16 \mathrm{c}$. p. lamp was found to be about 0.001 or 0.002 candle. Thus we see that not only would the relative error in comparing a $16 \mathrm{c}$. p. lamp with a 32 c. p. lamp be negligible, but that the absolute error for either one would be negligible in the most refined measurements, being of the order of magnitude of 1 part in 10,000. To be sure, the determination of this correction may be considerably in error, but since the correction is so very small it could be increased to several times its value and still be beyond recognition.

All of these measurements were made with the windows covered with heavy curtains, the only sources of illumination of the walls being lamps similar to ones that would ordinarily illuminate the walls when making photometric measurements. When the curtains were raised the effect was greatly increased, so that the error in a $32 \mathrm{c} . \mathrm{p}$. lamp was several tenths of a candle, varying with the brightness of the sky and with the distribution of objects about the room. Approximately the same relative error was found for the $16 \mathrm{c}$. p. lamp, so that in comparative measurements the errors in general would be negligible; but since the errors of measurement of these corrections were necessarily large, and since we found the corrections to vary considerably when an observer moved in front of a window, it is desirable to exclude daylight by curtains.

In conclusion, I desire to express my indebtedness to Mr. F. E. Cady for valuable assistance in making the observations. 


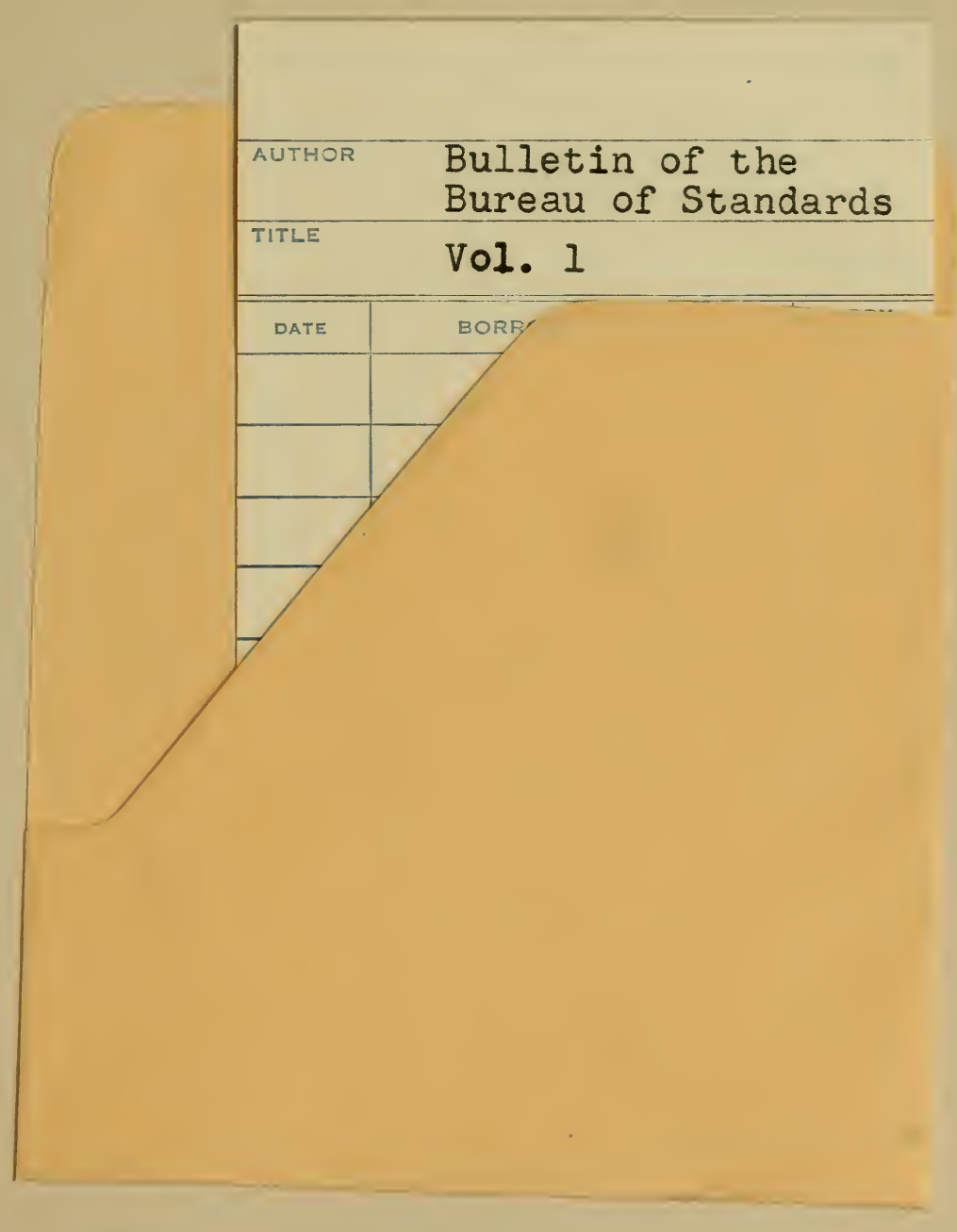




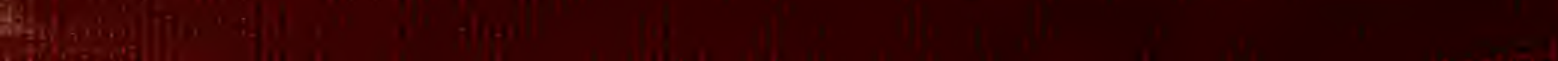

